210 MANAGEMENT OF PATIENTS WITH CONGENITAL TOXOPLASMA INFECTION FROM 1 TO 18 YEARS OF AGE

doi:10.1136/archdischild-2012-302724.0210

F Peyron, M Wallon. Service de Parasitologie, Hôpital de la Croix-Rousse, Lyon, France

At the age of 1 year congenital toxoplasmosis presents a wide spectrum of clinical signs ranging from patent neurological abnormalities to sub clinical infection. At this age treatment is usually discontinued as only cyst stage of parasites are present on which the currently used drugs are inefficient.

Patients with severe lesion, mainly hydrocephalus associated or not to visual impairment usually born from women who were not screened during pregnancy have to be placed in institution or have to be enrolled in special care programs.

Children treated ante and perinatally displayed a totally different presentation. In this setting gross abnormalities are very rare. In a cohort of 480 congenitally infected newborns we observed 5 hydrocephalus. Retinochoroiditis were observed in 8 cases. Ocular lesions are generally diagnosed later in life either because lesions are peripheral or because they occurred later, even after the age of 10 . Occurrences or relapses are usually unpredictable but age (6 and 11 years) or pregnancy appear to be periods of risk.

In such clinical settings congenital toxoplasmosis should be considered as a chronic ophthalmologic disease. Weather these patients should be regularly followed is debatable but for counselling pregnant women who seek for information about the long term outcome in their infants such systematic follow up is the only way for providing unbiased information. In a cohort of congenitally infected adults yearly checked since childhood, visual function and quality of life scored well and majority of participant endorsed long term ophthalmologic follow up.

\section{OUTCOME OF CONGENITAL TOXOPLASMA INFECTION IN YOUNG ADULTS}

doi:10.1136/archdischild-2012-302724.0211

'JG Garweg, ${ }^{2} \mathrm{M}$ Wallon, ${ }^{3} \mathrm{C}$ Binquet, ${ }^{4} \mathrm{~F}$ Peyron. 'Uveitis and Retinal Disease, Swiss Eye Institute, University of Bern, Rotkreuz, Switzerland; 'University of Lyon, Lyon; ${ }^{3}$ Epidemiology and Biostatistics, University of Dijon, Dijon; ${ }^{4}$ Parasitology, University of Lyon, Lyon, France

Background Ocular lesions represent the most frequently encountered clinical manifestation of congenital toxoplasmosis (CT). Thus they may be correlated with disease activity, functional damage and impact on quality of life (QoL).

Patients The Department of Parasitology, Hôpital de la CroixRousse, Lyon, France, represents a national reference centre for patients with CT. Beyond a European cohort of 430 children with treated CT, 130 presented retinochoroiditis (OT) during a median follow up period of more 10 years.

Results In our European cohort, OT was present at birth in only $10 \%$ of instances, but late manifestation beyond ten years of age has to be expected. After 6 years, $24 \%$ will manifest OT, increasing to $30 \%$ after ten years. Lesions in both eyes have to be expected in $30 \%$ of instances, but no child in our cohort had bilateral visual impairment. Not surprisingly, $\mathrm{QoL}$ assessment revealed that treated CT has little effect on the vision related QoL and general well being of the affected individuals. This strongly contrasts to outcomes reported from Southern American cohorts with CT where $80 \%$ of the merely untreated neonates show ocular manifestations early in life, $65 \%$ present bilateral lesions, and foveal involvement is present in $50 \%$, resulting in significantly lower QoL scores.

Conclusions People with OT in Europe show less severe clinical courses and functional damage resulting in better vision-related QoL than individuals living in Southern America, especially since these frequently have bilateral lesions and more recurrences. Close clinical follow up is warranted in any case with ocular involvement.

\section{MECHANICAL SUPPORT IN PEDIATRICS}

doi:10.1136/archdischild-2012-302724.0212

EM da Cruz. Pediatrics, Children's Hospital Colorado, University of Colorado Denver, School of Medicine, Aurora, CO, USA

ECMO is the implementation of the cardio-pulmonary bypass machine for prolonged periods of time to sustain systemic perfusion and gas exchange. This is an invaluable tool in the care of children with severe refractory cardio-circulatory and pulmonary failure, preferably in patients with potentially treatable and reversible cardiac disease. Development of the ECMO principles, although still not fully supported by evidence-based data, has allowed to progress from a salvage therapy to a more commonly used treatment to allow time for cardio-pulmonary recovery. Therefore, timely initiation of ECMO may impact prognosis. This has been the driving force followed by some centers that have continually available rapid sequence ECMO programs. Nevertheless, selection of the "appropriate" patients for ECMO remains a challenge, is continuously evolving and very institution-dependent. The principle of starting ECMO after failure of maximal medical therapy may be counterproductive. Decision to initiate ECMO in a cardiac patient, particularly after surgery often follows the instinctive judgment of the team. Literature suggests that early initiation of mechanical support in this patient population has been related to better outcomes and better hospital survival. Tendency to initiate ECMO in the early postoperative period or early throughout decompensation seeks to maintain adequate perfusion, to minimize ongoing myocardial insult and to enhance myocardial recovery. The latter may help create a favorable environment for myocardial recovery. Justification for ECMO initiation ought to be based on the patient's lack of capacity to properly perfuse his tissues; hence the need to identify and use early markers of tissue perfusion anomalies.

\section{PREVENTING PREMATURITY: USING HUMAN GENOMICS TO UNDERSTAND BIRTH TIMING}

doi:10.1136/archdischild-2012-302724.0213

\section{Muglia. Cincinnati Children's Hospital Medical Center, Cincinnati, OH, USA}

The composition of the biological clock defining the duration of human pregnancy remains a central question in reproductive biology. Our goal is to understand the molecular signals comprising this biological clock to prevent preterm birth. We have generated and analyzed mice with defects in prostaglandin biosynthesis, prostaglandin catabolism, circadian clock molecules, and oxytocin production in efforts to begin to define the key physiological pathways. These genetic studies in mice reveal essential functions for cyclooxygenase-1-generated prostaglandin F2 alpha for labor onset and the degrading enzyme hydroxyprostaglandin dehydrogenase in the maintenance of pregnancy. While these studies have elucidated the pathway for parturition timing in rodents, the findings have resulted in limited understanding for mechanisms of human parturition. In this presentation, data that genetic factors contribute to human preterm birth will be summarized. We have analyzed DNA from families with recurrent preterm birth and control families without preterm infants through genome-wide association and whole exome sequencing. These efforts have identified potentially interesting new contributors to birth timing from the maternal genome, such as the follicle stimulating hormone receptor gene, novel phospholipase isoforms, and pathways harboring rare, predicted deleterious mutations. New data regarding contributors to birth timing from the fetal genome will also be discussed. 